\title{
Research and Realization of Spread Model of Fire Accident of Prairie based on WRDS and CA
}

\author{
WANG Jian \\ Wuhan University of Technology \\ e-mail: ncwangjian1978@qq.com
}

\author{
Ma Shu-yue \\ Jiujiang University \\ e-mail: ncwangjian1978@qq.com
}

\begin{abstract}
Fire accident in grassland is a disaster which endangered the pastoral area of prairie grievously, and the spread of fire accident will cause inestimable financial loss and vast environmental destruction. If we can estimate the extended tendency of fire in pasture land accurately, it will exert a beneficial influence on controlling the fire accident in grassland. In this paper, we realized an advanced spread model of fire accident in grassland called WFDS-CA, which was based on the model of WFDS, combing the model cellular automata which were discrete in space and time.
\end{abstract}

Keywords-fire of prairie; cellular automata; model of spread; WRDS

\section{INTRODUCTION}

The grassland of Mongolia autonomous region in our country is the most serious grassland fire place, its takes place fire which number accounts for about $26 \%$ of the total number of national fire every year, and annual fire area of which is as much as $97 \%$ of the national total fire area, prairie fire causes great loss to pastoral economy especially the animal husbandry, threatening the people's personal property security of the pastoral areas ,and becomes influential and important interference factors to the grassland animal husbandry sustainable and stable development. Prairie fire is a very complicated natural phenomenon, many caused by natural factors and human factors, many factors can be integrated to construction of prairie fire spreading model [1-3], will be strong support to the fire forecast warning, rescue and decision-making when the fire break out. This paper combines WFDS model with cellular automatically, constructs a new prairie fire spreading model.

\section{WFDS FIRE SPREAD MODEL AND CELLULAR AUTOMATA}

Prairie fire includes the complex physical and chemical process, which involves many kinds of effect of different influencing factors, fire spread trend depends on the atmosphere, terrain, grassland vegetation and comprehensive effect. But because of the atmosphere, terrain, fuel conditions, and other complex changes, and considering the factor of safety, makes prairie fire study be difficult to carry on comprehensive repeated field test. Usually, prairie fire study bases on wild fire and urban land border zone fire research foundation.
In most studies, local fire spread speed is described as wind speed, terrain slope, humidity and the result of the interaction of the fuel characteristics. Because the fire itself which caused by the local environment change, many current model [4-7] does not predict fire transient state. This is because that for established fuel, wind and terrain conditions, fire etc, many influential factor is assumed constantly. Therefore, the local wind and fire and wind interaction prediction on the state of the fire prediction is of great significance.

A complete and comprehensive grassland fire model should give full consideration to the atmosphere, fire, plant fuel three component interaction [8]. In the actual research, in order to simplify the model, all kinds of studies highlighting have different emphases, produced different fire model. Such as Canada's FBPS model (Hirsch 1996), Australia's McArthur meters model (Nobel et al. 1980), American FARSITE (Finney 1998) BEHAVE model (Andrews 1986; Andrews et al. 2003), the National technical Standards Institute (National Institute of Standards and Technology, NIST) proposed WFDS (Wildland - urban Interface Fire Dynamics Simulator) Fire spread simulation experiment model and so on.

\section{A. WFDS wildfires model}

The US National technical Standards Institute (National Institute of Standards and Technology, NIST) proposed WFDS (Wildland - urban Interface Fire Dynamics Simulator) Fire spread simulation experiment model, are usually called WFDS model. The current WFDS model version of the behavior of the fire prediction get through the open prairie fire experiment examination, this is what got through the CSIRO model in Australia (Cheney et al. 1993; Cheney and Gould 1995). It is suitable for relatively simple grassland fire surface of the situation, the fuel in the flat area exists, because the fuel is approximate thermal thin, space evenly, then fire spread is mainly limited to horizontal direction.

The model spread process of search flame front (chart 1), the parameters associated with the a flame forward speed, fuel dry humidity and wind speed and direction, etc. $\mathrm{R}_{0}=\left(0.165+0.534 * U_{2}\right) * \exp \left(\left\{-0.859-2.036 * U_{2}\right\} / W\right) * \exp (-0.108 * M)$

in the type, $R_{0}(\mathrm{~m} / \mathrm{s})$ is the fire spread speed, $U_{2}(\mathrm{~m} / \mathrm{s})$ for distance ground 2 meters position wind, $W(m)$ for the fire spread the width of the striker, $M(\%)$ for the dry humidity of grass. 


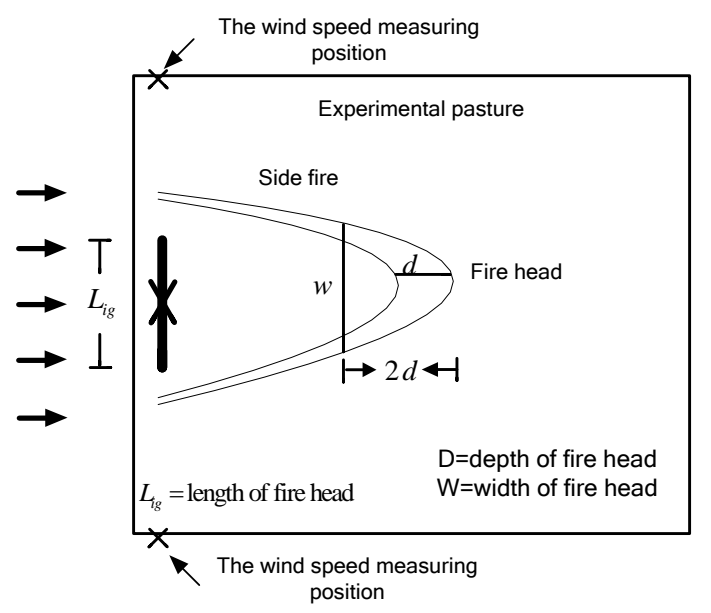

Figure 1. flame front schematic diagram

When the width of the flame front is big enough, it can simplify the type for the following form:

$$
\mathrm{R}_{s s}=\left(0.165+0.534 * U_{2}\right) * \exp (-0.108 * M)
$$

According to the prairie fire spreading principle and common sense, when the wind speed has no effect on prairie fire, prairie fire at a certain natural spread speed to approximate circular state to spread around, the speed of spread for:

$$
\mathrm{R}_{s s}^{\prime}=0.165 * \exp (-0.108 * M)
$$

\section{B. Cellular Automata}

Cellular Automata (Cellular Automata, CA) [9] is proposed and established by the American mathematician, also a famous computer scientist, Von Neumann . Von Neumann proposed the first selfreplicating cellular automata which was performed by twodimensional square grid composition, the thousands of basic yuan cell constitute ego is progenitive structure, each cellular has as many as 29 May state. Evolution rules depend on each celluar state and its four nearest neighbor celluar state.

With the cellular automata research theory studied deeply, cellular automata model has been had relatively successful application in all walks of life, and in the case of fire simulation problem it provides a set of effective methods, domestically, Chinese academy of sciences institute of geography professor jiancheng luo, who has also use cellular automata to simulate the spread of the forest fire, made the corresponding research and reasonable simulation. But as to relevant spread simulation research in the use of cellular automata to prairie fire, there is no explicit successful cases at home and abroad.

\section{PRAIRIE FIRE SPREADING MODEL BASED ON THE WFDS AND CA}

After the study, in the real prairie fire research analysis, we can divide the spread of fire into two partsr from essentially : one is when the fire occurs, combustion medium combustion status, specific to prairie fire, is the grassland vegetation's own combustion condition; The second is when a fire happens in flames, and the movement trend, this will decide a fire finally too far regional forecast, if we can accurately simulate the fire flame movement trend, we will be able to provide more effective before a disaster early warning, the disaster relief and post-disaster placement and auxiliary decision-making function.

Combustion medium's own combustion condition, we call combustion essential factors, it is the foundation of what we research in the fire burning movement trend, over the course of the study, we find that, the influence factors of combustion nature, these parameters, such as vegetation type, fire ground wind speed and so on, can better fit with the various parameters in NIST - WFDS model, on the other hand, CA model has the natural space and time discreteness, as the research fire burning movement trend to provide effective tools, in fire, obstacles, terrain slope, wind direction and other factors all can effective abstraction to CA model, coupled with NIST - WFDS successfully got the result of burning nature factors, through the combination of WFDS and CA can construct a reasonable prairie fire spreading model.

\section{A. The fire speed in the model}

According to the NIST - WFDS equations, comprehensive consideration of the different types of grass on final comburent influence, in the WFDS rotor forward velocity formula, add the different type of grass impact factor, the rotor forward velocity formula is as follows:

$$
R_{h}=M *\left(0.615+0.534 * U_{W}\right) * \exp (-0.108 * H)
$$

$R_{h}$ means rotor speed, the unit $\mathrm{m} / \mathrm{s}, U_{W}$ means the wind speed of ground 2 meters, the unit $\mathrm{m} / \mathrm{s}, H$ means dry humidity of the comburent, the unit $\%, M$ means different type of grass impact factor;

Through this formula, we can get a prairie fire process rotor speed value, and is convenient to load the rotor speed into the cellular automata model to further study the spread of the prairie fire trend.

\section{B. The model spread trend}

Target grasslands get through the grid chemical processing, generates two dimensional cellular (Fig .2)which accord with cellular automata rules, according to cellular evolution rules and the following operation formula, we can get in setting long fire spread data.

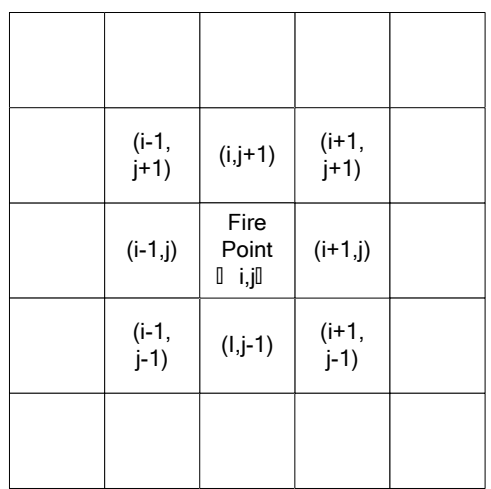

Figure 2. cellular grid after spheroidizing treatment 
Definition of a single cell $S(I, j)$ in $t$ time for combustion state is $S_{i, j}^{t}$, its value is a single intracellular burning area and single cell area ratio:

$$
S_{i, j}^{t}=\frac{A_{t}}{A}, S_{i, j}^{t} \text { means a single cell } \mathrm{S}(\mathrm{I}, \mathrm{j}) \text { in } \mathrm{t}
$$

moment combustion state value, $A_{t}$ means a single intracellular burning area, ${ }^{A}$ means a cell area;

In which,

$$
S_{i, j}^{t+1}=f\left(S_{i, j}^{t}, S_{i-1, j}^{t}, S_{i, j-1}^{t}, S_{i+1, j}^{t}, S_{i, j+1}^{t}, S_{i-1, j-1}^{t}, S_{i+1, j-1}^{t}, S_{i-1, j-1}^{t}, S_{i+1, j+1}^{t}\right)
$$

$$
S_{i, j}^{t}, S_{i-1, j}^{t}, S_{i, j-1}^{t}, S_{i+1, j}^{t}, S_{i, j+1}^{t}, S_{i-1, j-1}^{t}, S_{i+1, j-1}^{t}, S_{i-1, j-1}^{t}, S_{i+1, j+1}^{t},
$$

respectively means a single cellular $S(I, j)$ itself in $t$ moment combustion state and its adjacent eight single cellular in t moment combustion state; Specific calculus formula is shown below:

$$
S_{i, j}^{t}=S_{i, j}^{t}+\left(w^{*} S_{i-1, j}^{t}+S^{*} S_{i, j-1}^{t}+E^{*} S_{i+1, j}^{t}+N^{*} S_{i, j+1}^{t}\right)+0.78^{*}\left(W S^{*} S_{i-1, j-1}^{t}+E S * S_{i+1, j-1}^{t}+W N^{*} S_{i-1, j-1}^{t}+E N^{*} S_{i+1, j+1}^{t}\right)
$$

Which inside, $S_{i, j}^{t+1}$ indicates a single cellular S (I, j) in $\mathrm{t}+1$ time of combustion state, $S_{i, j}^{t}$ indicates a single cellular $\mathrm{S}(\mathrm{I}, \mathrm{j})$ in $\mathrm{t}$ moment combustion state, $S_{i-1, j}^{t}$ indicates a single cellular $S(I, j)$ in the west a single yuan cell in t moment combustion state, ${ }^{t}{ }_{i, j-1}$ indicates a single cellular $S(I, j)$ in the south of a single yuan cell in $t$ moment combustion state, $S_{i+1, j}^{t}$ indicates a single cellular $S(I, j)$ east single yuan cell in t moment combustion state, $S_{i, j+1}^{t}$ indicates a single cellular $\mathrm{S}(\mathrm{I}, \mathrm{j})$ in he north of the single yuan cell in t moment combustion state, $S_{i-1, j-1}^{t}$ indicates a single cellular $\mathrm{S}(\mathrm{I}, \mathrm{j})$ in the southwest single yuan cell in t moment combustion state, $S_{i+1, j-1}^{t}$ indicates a single cellular $S(I, j)$ in the southeast of single yuan cell in t moment combustion state, ${ }^{t}{ }_{i-1, j+1}$ indicates a single cellular $S(I, j)$ in the northwest of single yuan cell in $t$ moment combustion state, $S_{i+1, j+1}^{t}$ indicates a single cellular $S(I, j)$ in the northeast of the single yuan cell in $\mathrm{t}$ moment combustion state, E, S, N, W, ES, EN, WS, WN, means east, south, west, north, southeast and northeast, southwest, northwest to influence factor.

\section{C. the realization of the model program}

This paper bases on the study of the WFDS - CA prairie fire spreading model, combines with GIS based software, realizes the prairie fire spread simulated dynamic display, data statistic analysis function. Part of the program flow diagram of prairie fire spread is as shown in Fig .3:

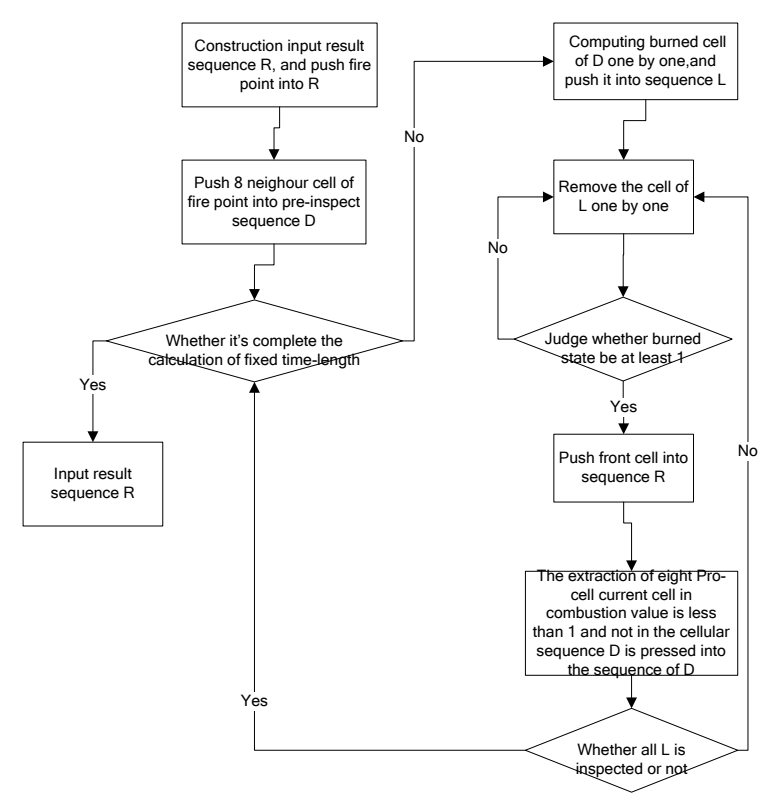

Figure 3. the program flow diagram of prairie fire spread

Finally through the SuperMap iServer2008R2 platform we realized running effect of the WFDS - CA prairie fire spreading model, as shown in Fig .4:

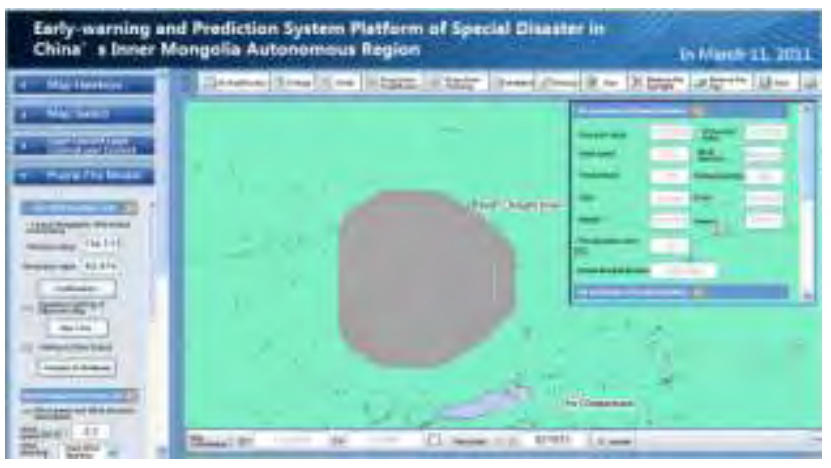

Figure 4. WFDS - CA prairie fire spreading model rendering operation 


\section{CONCLUSION}

Combined the NIST-WFDS model with cellular automata model to simulating predict the spread of the prairie fire,deeply combines the two kinds of models and grassland vegetation characteristics,and studies the main factors that influence grassland fire speed in the specific circumstance,takes the fire speed into cellular automata model for further research in grassland fire spread trend. fire speed into cellular automata model for further research in grassland fire spread trend, get WFDS - CA prairie fire spreading model, and with the help of SuperMap iServer platform program to realize the model, get a more satisfactory results through the operation.However, because of the complexity of the grassland fire burning, it still needs further study for grassland fire burning velocity and the spread between the trend of complex connection, and through a lot of prairie fire historical data to correct prairie fire spreading model, we expect to get the most accurate model, and can be applied in real life,to deal with prairie fire to provide corresponding support and help for the government or as a footnote.

\section{REFERENCES}

[1] William Mell, Mary Ann Jenkins. Physics-Based Approach to Modeling Grassland Fires.International Journal of Wildland Fire ,January 19, 2007.
[2] Yuewen Liu, Hongye Ynag, Shuo Wang. Multi-Factor Spread Simulation of Forest Fire Based on CA Model. Proceedings - 2009 International Conference on New Trends in Information and Service Science, NISS 2009 . 1087-1089. 2009.

[3] Hongye Yang, Chun Zhao, Yuewen Liu. Gis-based inner Mongolia grassland fire spread simulation system. International Conference on Computer Science and Software Engineering, CSSE 2008. V3,923-925.2008.

[4] Kevin McGrattan, Simo Hostikka, Jason Floyd. Fire Dynamics Simulator Technical Reference Guide. NIST Special Publication 1018-5.

[5] YueXiuQuan, zhou daowei, JiangShiCheng. The hulunbuir prairie fire analysis $[\mathrm{J}]$. Journal of northeast normal university natural science edition, 1999, no.4, 111-116.

[6] ZhouWeiqi, WangShiXin, ZhouYi, ZhaoQing. Grassland fire rating forecast [J]. Journal of natural disasters, 2004, 13 (2), 75 - 79.

[7] Suehe, LiuGuixiang. Prairie fire monitoring system and its application [J]. China grass, 1996 the fifth period, 66 - 69 .

[8] LiuYueWen, YangHongYe, Wangshuo, ZhaoChun. A based on $\mathrm{CA}$ of forest fire spread simulation model design and implementation in Inner Mongolia region - for example. Science of disaster. The 3 rd issue in 2009.

[9] zhouchenghu, SunZhanLi, thanks a spring. Geographic cellular automata research. Science press, Beijing, 1999

[10] Suehe, LiuGuixiang. Prairie fire monitoring system and its application [J]. China grass, 1996 the fifth period, 66 - 69. 